Cahiers « Mondes anciens »

ANCIENS

Histoire et anthropologie des mondes anciens

$10 \mid 2018$

Les politiques familiales dans les mondes antiques

\title{
Entre discours officiels et pratiques privées
}

Aspects du droit familial mésopotamien au $\mathrm{II}^{\mathrm{e}}$ millénaire avant n.è.

Family in Ancient Near-East. Between Official Discourse and Private Practices

\section{Sophie Démare-Lafont}

\section{(2) OpenEdition}

Journals

Édition électronique

URL : http://journals.openedition.org/mondesanciens/2036

DOI : 10.4000/mondesanciens. 2036

ISSN : 2107-0199

Éditeur

UMR 8210 Anthropologie et Histoire des Mondes Antiques

Référence électronique

Sophie Démare-Lafont, "Entre discours officiels et pratiques privées », Cahiers " Mondes anciens » [En ligne], 10 | 2018, mis en ligne le 31 janvier 2018, consulté le 19 avril 2019. URL : http://

journals.openedition.org/mondesanciens/2036 ; DOI : 10.4000/mondesanciens.2036

Ce document a été généré automatiquement le 19 avril 2019

\section{cc) $($ ) $\odot \odot$}

Les Cahiers «Mondes Anciens » sont mis à disposition selon les termes de la licence Creative Commons Attribution - Pas d'Utilisation Commerciale - Pas de Modification 4.0 International. 


\title{
Entre discours officiels et pratiques privées
}

\author{
Aspects du droit familial mésopotamien au $\mathrm{II}^{\mathrm{e}}$ millénaire avant n.è. \\ Family in Ancient Near-East. Between Official Discourse and Private Practices
}

\section{Sophie Démare-Lafont}

Dresser un portrait de la famille proche-orientale s'apparente à une technique qu'on qualifierait de pointilliste en peinture: l'historien ne peut procéder que par petites touches et l'image d'ensemble qu'il parvient à dessiner reste assez floue, comme tremblée. L'iconographie n'est pas d'une grande aide, qui représente rarement les couples et un peu plus souvent les enfants sur les genoux de leur nourrice, ou moins probablement de leur mère (Parayre 1997, p. 67-73). Du côté des textes, l'absence d'exposé théorique sur ce thème (comme sur tous les autres) contraint l'interprète moderne à restituer ce qu'il croit comprendre des informations concrètes livrées par les tablettes.

Clichés et poncifs anciens teintés d'orientalisme ont parfois donné l'illusion de dissiper les incertitudes à coups de préjugés et d'anachronismes: polygamie généralisée, innombrable descendance, droit de vie et de mort du père de famille sur sa maisonnée, ou encore incapacité totale de la femme, autant de situations qui, certes, sont attestées ponctuellement mais ont pu être érigées en principes généraux du seul fait des lacunes de nos sources (Démare-Lafont 2015 ; Stol 2016).

3 Il est vrai que la documentation écrite elle-même livre des données très disparates. Les textes juridiques fournissent une information à la fois abondante et incomplète. Les recueils législatifs (Roth 1997) abordent les principaux aspects du droit familial (mariage, divorce, filiation, successions) sans pour autant brosser un tableau d'ensemble de la matière, conformément à la méthode casuistique des législateurs orientaux. Les contrats et procès, relativement peu nombreux au regard de la longue durée de l'histoire mésopotamienne, décrivent des situations particulières qui ne sont peut-être pas représentatives des pratiques courantes. 
Hors des sources proprement juridiques, les lettres se font principalement l'écho des disputes et contentieux divers, qui tournent souvent autour de questions d'héritage ou d'affaires économiques. La littérature mythologique ou sapientiale donne des éclairages partiels mais assez évocateurs, qui prennent parfois une dimension pédagogique. Tel est le cas notamment de l'histoire du mariage du dieu Enlil (Zgoll 2013), racontée en deux versions qui correspondent respectivement à « ce qu'il faut faire » et « ce qu'il ne faut pas faire » : pour fonder un foyer, il faut faire sa demande en bonne et due forme et obtenir le consentement des parents de la jeune fille, qui est généralement très jeune, voire une enfant. Dans le « mauvais » scénario au contraire, le dieu attire sa proie hors de chez elle et la viole puis la quitte, ce qui déclenche une longue et pathétique errance de la victime à la poursuite de son bourreau, chaque rencontre étant ponctuée de grossesses clandestines puisque hors mariage. Le rapprochement de ces deux récits fait ressortir un discours moralisant sur les bienfaits de la famille légitime et les dangers de l'amour libre.

On s'attendrait donc à trouver dans la littérature politique et officielle une reprise de ces thèmes, et ce d'autant plus que la famille est à la fois le modèle et le socle sur lequel s'appuie le pouvoir monarchique, forme de gouvernement la mieux connue pour le monde proche-oriental ancien (bien qu'elle ne soit pas la seule). Au II millénaire avant n. ̀̀., le palais fonctionne comme une grande maison et son organisation n'est guère différente de celle des villas et autres belles demeures des élites politiques et administratives (Westbrook 2000, p. 28-30). Les dignitaires locaux mélangent d'ailleurs souvent la gestion de leurs biens de fonction avec celle de leurs fortunes propres, montrant ainsi la continuité des méthodes et des interlocuteurs.

Mais si les patrimoines obéissent aux mêmes règles, quoiqu'à des échelles différentes, dans les sphères publique et privée, la famille en tant que telle est absente de la rhétorique royale telle qu'on peut la lire notamment dans la littérature politique officielle. Certaines figures comme celles de la veuve et de l'orphelin sont cependant récurrentes dans les prologues et les épilogues des codes de lois (voir infra) et servent d'appui à l'apologie du gouvernement royal pour convoyer des valeurs comme la bienveillance et la justice sociale.

7 La moralité et les bonnes mœurs sont également évoquées dans la législation royale, à travers notamment la répression de l'adultère ou de l'inceste (Démare-Lafont 1999, p. 29-91 et 173-236). Plus exceptionnellement, le roi et le juge interviennent pour interdire à des jeunes gens de bonne famille leurs mauvaises fréquentations (DémareLafont 2011).

Cependant, pour permanents qu'ils soient, ces thèmes ne trouvent quasiment pas d'échos dans l'action politique du roi sur le terrain. Pour donner une réalité et une consistance à ces motifs littéraires, il faut se tourner vers les actes de la pratique, qui montrent comment fonctionnent concrètement les mécanismes de protection de la famille, qu'il s'agisse des biens ou des personnes. Ces exemples illustrent les techniques imaginées par les particuliers pour prendre en charge les aléas de l'existence et mettre en œuvre les solidarités entre générations.

9 Mis bout à bout, ces éléments recueillis dans les sources montrent que, s'il y a une politique familiale au Proche-Orient ancien, elle s'appuie sur deux aspects complémentaires qui sont l'affichage idéologique d'un côté et l'engagement pragmatique de l'autre. 


\section{La famille dans le champ du discours officiel}

10 Parmi les thèmes qui reviennent constamment dans les inscriptions royales et la littérature officielle, il y a la piété du souverain, son courage au combat et sa capacité à rendre la justice. Ce dernier thème est abondamment développé, notamment dans le célèbre Code de Hammurabi (vers 1750 avant n. è.), qui présente le roi comme le juge naturel de ses sujets, sorte de « fontaine de justice » toujours disponible et accessible.

C'est ce qu'on peut lire notamment dans ce passage de l'épilogue :

Que l'homme lésé qui a un procès aille devant ma statue de roi de justice, qu'il lise ma stèle inscrite, qu'il écoute mes paroles très précieuses et que ma stèle lui révèle son procès, de sorte qu'il voie la sentence qui le concerne et qu'il s'apaise (en se disant) : « Hammurabi, le seigneur, qui est comme un vrai père pour le peuple, s'est soucié de la parole du dieu Marduk son seigneur et a assuré partout la victoire de Marduk. Il a contenté le cœur de Marduk son seigneur et assuré pour toujours le bonheur des gens et fait régner la justice dans le pays » (xlviii 3-38; transcr. Roth 1997, p. 134).

Il est frappant de constater que le roi se présente ici comme un père pour ses sujets, parce qu'il est capable de les protéger contre les injustices et de faire respecter sur terre l'ordre divin voulu par Marduk, le dieu de Babylone (Charpin 2013, p. 364).

La fonction paternelle s'incarne donc dans le souverain à travers ce pouvoir de juger, qui rappelle la juridiction domestique du chef de famille, souvent considérée comme une puissance arbitraire et illimitée. Or, la justice royale telle qu'elle est décrite dans le texte ne s'exerce pas de manière discrétionnaire mais sur la base de règles écrites et même publiées (au moyen de la stèle), donc opposables. De la même manière, les membres de la maisonnée ne sont pas livrés à la tyrannie aveugle du patriarche, dont les prérogatives sont limitées par la loi, et incluent certains châtiments corporels sans aller jusqu'au droit de tuer. On sait ainsi que le mari assyrien pouvait frapper sa femme, la fouetter, lui tirer les cheveux ou abîmer ses oreilles (Lois assyriennes tablette A §§ 57-59; Cardascia 1969, p. 255-260 ; Démare-Lafont 2012). En cas de vol, il pouvait lui couper les oreilles ou le nez (Lois assyriennes tablette A §§ 4-5; Cardascia 1969, p. 99-103; Démare-Lafont 2012). Quoique cette sévérité soit sans équivalent dans les autres législations mésopotamiennes, elle ne comporte pas la faculté de mettre à mort l'épouse, les enfants ou les esclaves. Le crime passionnel lui-même est soumis au contrôle du juge ( $\S 15$ tablette A Lois assyriennes ; Cardascia 1969, p. 119-123 ; Démare-Lafont 2012), et ne s'apparente donc pas à une prérogative du mari.

14 Une autre occurrence de la figure paternelle à propos du monarque concerne la vie religieuse, en particulier dans le domaine des cultes funéraires. L'une des obligations familiales, qui conditionnait la vocation successorale, consistait à honorer la mémoire des défunts au cours de cérémonies consistant en offrandes alimentaires accompagnées d'une litanie des noms des morts. Ne pas accomplir ces rites était ressenti comme une forme de damnatio memoriae, une malédiction qui effaçait l'individu et son lignage de la mémoire collective. C'est pour pallier cet inconvénient que les couples stériles ou inquiets pour leur descendance adoptaient un enfant ou un adulte, dont la mission explicite était d'enterrer leurs parents et de veiller au respect des traditions funéraires (voir infra).

Mais malgré toutes ces précautions, il pouvait arriver qu'un individu mourût sans postérité. C'était alors au roi d'invoquer leur souvenir, comme le montre la formule 
insérée dans la prière adressée aux ancêtres du roi babylonien Ammi-șaduqa (1646-1626), qui invite au banquet funéraire «tout être humain, de l'Est à l'Ouest, qui n'a personne pour prendre soin de lui » (Charpin 2013, p. 364). Ceux qui n'ont plus de famille sont ainsi associés au lignage royal, au même titre que les soldats morts au combat, le souverain régnant devenant pour eux une sorte de sauveur providentiel, un père de substitution.

C'est dans ce même esprit que le roi offre sa sauvegarde, au moins théorique, aux veuves et aux orphelins, tous deux privés de protection familiale et donc particulièrement vulnérables. Dès le milieu du $\mathrm{III}^{\mathrm{e}}$ millénaire avant n. è., le roi sumérien Urukagina proclama une amnistie générale, couvrant notamment les débiteurs insolvables, afin dit-il « de ne pas livrer au riche la veuve et l'orphelin » (Frayne 2007, p. 265). Ce leit-motiv du discours politique oriental est repris à la fin du III ${ }^{e}$ millénaire par le prince sumérien Gudea de Lagaš (Edzard 1997, p. 36 et 98) puis par le roi sumérien Ur-Namma, qui affirme dans le prologue de son code qu'il n'a pas "livré l'orphelin au riche (ni) la veuve au puissant» (Roth 1997, p.16), et se retrouve également dans l'épilogue du Code de Hammurabi où les lois sont présentées comme un effort « pour que le fort n'opprime pas le faible, pour faire justice à l'orphelin et à la veuve» (Roth 1997, p. 133). L'image est reprise dans la Bible, au Code de l'Alliance (Ex. 21, 22-23), assortie d'une menace à caractère talionique puisque la sanction divine contre l'oppresseur ferait de sa femme une veuve et de ses enfants des orphelins.

Il est vrai que les illustrations de la bienveillance royale à l'égard de ces catégories particulières de démunis sont introuvables dans la documentation cunéiforme. La Bible offre un cas de recours au souverain à travers l'histoire de la femme de Téqoa (2 Sam. 14, 4-11), qui, déguisée en veuve, demande audience au roi David pour implorer sa miséricorde : elle le supplie d'intervenir pour stopper la vengeance décrétée par le clan contre l'un de ses fils, meurtrier de son frère. La réponse favorable de David, qui s'engage personnellement à défendre la vie du fautif, montre que le souverain est attentif à la cohésion des familles et prétend imposer son autorité contre celle du lignage, afin de respecter sa mission de secours aux veuves et aux orphelins. On sait que l'affaire est en réalité une parabole pour inciter David à rappeler son fils Absalom, en fuite après le meurtre d'Amnon. L'épisode sert donc de prétexte pour célébrer les qualités du roi juste, seul capable d'assurer la protection de l'institution familiale.

Reste que dans ce qu'on pourrait appeler «la vraie vie », les choses se passent autrement, sans intervention royale, et ce sont les solidarités lignagères ou les montages juridiques audacieux qui tiennent lieu de politique familiale.

\section{Solidarités familiales et pratiques juridiques}

Les actes de la pratique mésopotamiens montrent divers types d'arrangements contractuels qui permettent de garantir la survie des membres les plus vulnérables de la famille.

L'exemple des veuves est très emblématique à cet égard. Elles se définissent au ProcheOrient ancien comme des femmes ayant perdu non seulement leur époux mais aussi leur père et leurs frères (Roth 1991, p. 3), ce qui les place dans une grande précarité car vis-àvis de leur belle-famille, elles sont considérées comme des étrangères.

Pour faire face à cette situation, elles peuvent soit se remarier, soit bénéficier d'une libéralité du mari. 

testateur transforme sa femme en "père et mère" de sa maison, ce qui revient techniquement à opérer une sorte de donation au conjoint survivant. La veuve a donc l'usufruit viager de la totalité du patrimoine, ce qui l'autorise notamment à demeurer dans la maison familiale sans pouvoir en être expulsée par ses enfants, lesquels sont tenus de pourvoir à son entretien sous peine d'être privés de leur part successorale. Le testament suivant, provenant de la ville syrienne d'Emar (non loin d'Alep) et daté du XIV s. avant n. è., en apporte une illustration :

Muhra-ahi, fils d'Abi-Ra, de son vivant, a fait siéger ses frères et a fixé le destin de sa maison, de ses enfants et de son épouse. Il a dit: «Voici que la fille de Kaga est mon épouse. Elle est père-et-mère de ma maison. Tant qu'elle vivra, elle demeurera dans la maison principale. Personne ne revendiquera contre elle.

Et Rašap-kabar son fils l'entretiendra. Si Rašap-kabar n'entretient pas son père-et- 
mère, il n'aura rien de sa maison. Et si Igmulu entretient aussi son père-et-mère, alors la moitié du kirșitu sera sa part successorale. S'il ne l'entretient pas, il n'aura rien de la moitié du kirșitu constituant sa part successorale. » Noms de 4 témoins ; date (Arnaud 1986, nº 15, p. 23-24).

Si le mari n'avait rien prévu, la communauté pouvait procurer une activité rémunérée à la femme, et ainsi contribuer, quoique modestement, au coût de son entretien, en attendant qu'un membre de sa belle-famille propose de l'épouser. C'est ce que prévoit le droit assyrien au milieu du II $^{\mathrm{e}}$ millénaire avant $n$. è., pour venir en aide notamment à la femme de l'absent, qui est dans une situation assez comparable à celle de la veuve.

Si une femme a été donnée (en mariage) et l'ennemi a pris son mari, qu'elle n'a pas de beau-père ni d'enfant, elle attendra son mari pendant deux ans. Pendant ces deux années, s'il n'y a pas de quoi manger, elle ira (le) déclarer. Si elle est une dépendante du palais, son [...] la nourrira et elle travaillera pour lui. Si elle est l'épouse d'un soldat-hupšu, [...] la nourrira [et elle travaillera pour lui] (§ 45 Lois assyriennes tabl. A ll. 103-108; transcr. Roth 1997, p. 170-171).

Il arrive aussi, toujours dans ces mêmes régions, que le père assigne à sa fille une double identité masculine et féminine, pour lui transmettre l'obligation d'accomplir les rites funéraires. Tel est le cas dans cet autre testament d'Emar ( $\mathrm{XIV}^{\mathrm{e}} \mathrm{s}$. avant $\mathrm{n}$. è.) :

À compter de ce jour, Muzazu fils de Šamana, de son vivant, a fixé le destin de sa maison. Il a dit: "Voici que Hebate mon épouse est père-et-mère de ma maison. Voici que j'ai établi Al-hati ma fille comme femme-et-homme. Elle invoquera mes dieux et mes morts. Voici que j'ai donné mes immeubles et mes meubles et tout ce qui m’appartient à Al-hati ma fille.

Si mon épouse Hebate suit un étranger, elle devra poser son vêtement sur le tabouret et aller où elle voudra. Si ma fille Al-hati meurt et n'a pas de descendance, son mari Ahu-yaqaru pourra prendre une autre épouse. Les enfants qu'elle enfantera, avant et après, sont mes enfants. Et si Al-hati et Ahu-yaqar meurent et qu'ils n'ont pas d'enfants, Al-ummi et Panu hériteront leurs biens. »

Noms de huit témoins (Huehnergard 1983, n² 2 p.18-19; Tsukimoto 1991, n² 26, p. 289).

31 En l'occurrence le testateur ne semble pas avoir de fils et ne souhaite pas en adopter, préférant manifestement s'en remettre à ses futurs petits-enfants. Les pères avaient parfois recours à cette fiction alors même qu'ils avaient des fils (e.g. Arnaud 1986, n 181 p. 194-195), dont on suppose qu'ils étaient trop jeunes ou peut-être en mauvaise santé, ce qui laissait craindre une extinction de la lignée.

On retrouve ici le souci, déjà évoqué plus haut, de garder le souvenir des défunts à travers les cultes familiaux, qui incombent théoriquement à un fils, peut-être à l'aîné. La fille peut y être tenue en vertu de la volonté paternelle, au prix d'un montage audacieux, qui remet en perspective la question très actuelle du genre. Cette question a été abondamment analysée notamment par B. Lion $(2007,2009,2011)$ et trouve des prolongements intéressants dans les récits miraculeux de la région du Tur Abdin à l'époque contemporaine (Briquel-Chatonnet 2010).

Des solutions plus classiques sont également attestées, qui ont pour finalité principale d'apporter un soutien matériel aux ascendants lorsqu'ils deviennent trop âgés pour travailler. La documentation d'époque paléo-babylonienne (première moitié du $\mathrm{II}^{\mathrm{e}}$ millénaire avant n.è.) offre ainsi de nombreux contrats par lesquels les fils, et plus rarement les filles, s'engagent à fournir à leur père des rations annuelles d'orge, d'huile et de laine, correspondant aux trois denrées de base nécessaires à la vie quotidienne. Il peut s'agir d'enfants biologiques ou adoptés, comme le montrent les deux exemples suivants. 
Le premier, provenant de Babylonie et datant de 1749 avant n.è., est une convention d'entretien d'un père par ses trois fils :

1000 litres d'orge, 2 kilos de laine et 2 litres d'huile que Damqum donnera à Abuwaqar son père ; 1000 litres d'orge, 2 kilos de laine et 2 litres d'huile qu'Apil-Marduk donnera à Abu-waqar son père ; 1000 litres d'orge, 2 kilos de laine et 2 litres d'huile que Šamaš-magir donnera à son père. D'un commun accord, ils lui ont assigné (ces rations). Il ne (les) changera pas. Ils ont juré par le roi. Noms de 5 témoins. Ils lui donneront (les rations) le $4^{\mathrm{e}}$ mois au $30^{\mathrm{e}}$ jour échu. Date. Sceau (Anbar et Stol 1991, $\mathrm{n}^{\circ} 22$, p. 34-35).

Le second document est un contrat d'adoption de Nippur (sud mésopotamien) du XVIII ${ }^{\mathrm{e}} \mathrm{s}$. avant n. è. :

Ilabrat-tayyar a pris Patiya en qualité de fils. Maison, champs, verger autant qu'il y en a, Ilabrat-tayyar (les) a donnés à Patiya son fils. Si Patiya dit à Ilabrat-tayyar son père : « Tu n'es pas mon père ", il payera $1 \frac{1}{2}$ mine d'argent. Et si Ilabrat-tayyar dit à Patiya son fils : « Tu n'es pas mon fils », il payera [1/2 ?] mine d'argent et il perdra sa maison et tous ses biens. Patiya payera chaque mois à Ilabrat-tayyar une ration annuelle de 300 litres d'orge, [n] kilos de laine et 6 litres d'huile (PBS 8/2 153; Stone et Owen 1991, n 5, p. 42-43).

Dans les deux cas, ils ont très certainement reçu par anticipation leurs parts successorales, qu'ils détiennent souvent en indivision, et ils assument solidairement la charge financière de leur père désormais privé de ressources.

Une variante consiste à promettre l'affranchissement à un ou une esclave de la maison après le décès du maître, à condition de l'avoir entretenu sa vie durant. C'est ce que prévoit ce contrat de Nippur du XVIII ${ }^{\mathrm{e}} \mathrm{s}$. avant $\mathrm{n}$. è. :

Ur-dukuga a libéré [Unetelle sa servante]. Il a purifié son front et a dressé pour elle un document concernant sa libération (litt. " purification »). Tant qu'il vivra, elle le servira. Ur-dukuga le père, de son vivant, a juré par le roi que, lorsque Ur-dukuga sera mort, les héritiers d'Ur-dukuga ne revendiqueront pas à propos de son statut de servante. Nom d'un témoin (3N-T 845 ; Roth 1979, p. 108-109; Westbrook 1995, p. 1650 n. 55).

Concrètement, la vie de l'esclave reste la même, mais juridiquement, sa relation change puisqu'il/elle n'agit plus dans le cadre d'un statut mais d'un contrat, d'où la clause rendant ce changement opposable aux héritiers.

Il existe enfin des formes plus inattendues de solidarité entre débiteurs et créanciers qui imitent le modèle familial pour faire face à la paupérisation endémique qui traverse tout le $\mathrm{II}^{\mathrm{e}}$ millénaire av. n.è.

Une première solution consiste pour le débiteur à adopter un tiers qui payera sa dette et se remboursera sur la succession. C'est ce que décrit une tablette d'adoption de Nippur, dans laquelle un nommé Ipquša adopte un certain Ea-tayyar qui, le même jour, rembourse la dette de l'adoptant, ce qui conduit à réviser les modalités de sa succession : elle sera désormais partagée entre le fils biologique et le fils adoptif :

Ipquša fils de Dingir-kuta a pris Ea-tayyar fils de Ku... en qualité d'héritier. Le jour de l'adoption, Ea-tayyar a remboursé 1200 litres d'orge (représentant la) dette d'Ipquša son père. Du vivant d'Ipquša le père, Ea-turam le fils de son épouse, et Eatayyar le fils pris [en adoption] ont fait un nouveau partage des parts successorales. Une maison de $70 \mathrm{~m}^{2}$ à côté de celle d'Ipqu-Ea, fils de Dingir-kuta, $3600 \mathrm{~m}^{2}$ de champ dans le domaine d'Enlil-gara, à côté de celui d'Ipqu-Ea, la moitié de la maison autant qu'il y en a, c'est la part successorale d'Ea-turam. Une maison de $70 \mathrm{~m}^{2}$ à côté de celle d'Ea-turam son frère, $3600 \mathrm{~m}^{2}$ de champ dans le domaine d'Enlil-gara à côté de celui d'Ea-turam son frère, la moitié de la maison autant qu'il y en a, c'est la part 
successorale d'Ea-tayyar son frère. Ea-turam et Ea-tayyar fourniront chacun à Ipquša leur père 500 litres d'orge, 1,5 kilo de laine et 3 litres d'huile tous les ans. Celui d'entre eux qui ne lui fournira pas ces denrées perdra son statut d'héritier. Ils ont juré ensemble par le roi (BE 6/2 28 ; Stone et Owen 1991, $n^{\circ} 4$ p. 41-42). biens et ne semble donc pas insolvable. Le but est sans doute pour Ipquša de « doubler » son fils biologique, trop jeune ou malade, avec un héritier en âge de travailler et en bonne santé, qui pourra l'entretenir et assurer les rites funéraires à sa mort.

Derrière la sécheresse des clauses contractuelles, il y a souvent des histoires humaines pathétiques, liées aux périodes de crise que les tablettes appellent la « détresse » (dannūtu ), terme qui évoque à la fois la guerre et la famine, deux fléaux qui poussent les parents à vendre leurs enfants ou les débiteurs ruinés à s'engager au service de leur créancier. avant n. è.) dans lequel un débiteur, Yašar-da'i, se place dans la domesticité de Bulalu son créancier, qui est aussi paradoxalement son bienfaiteur puisqu'il l'a «fait vivre », ce qui signifie qu'il l'a sauvé d'une mort certaine par inanition :

Yašar-da'i, fils d'Aštartu-lit, a dit : «Pendant l'année où les hordes assiégeaient la ville, qu'un litre d'orge valait un sicle d'argent, je me suis endetté pour 1000 litres d'orge envers Bulalu, fils d'Arwu. Et voici que Bulalu m'a arraché à la famine et m'a fait vivre. » Et si à l'avenir, Yašar-da'i veut sortir de la maison de Bulalu, il devra donner à Bulalu un esclave à sa place. S'il fournit un esclave pour sortir de la maison, il devra le donner à Bulalu (et) il pourra aller où il veut. Mais s'il prend un esclave pour quelqu'un d'autre, cette tablette le confondra. Noms et sceaux de quatre témoins (TS 25 ; Démare-Lafont 2014, p. 399-400).

La forme que prend cette aide est a priori choquante dans nos cultures modernes. Pourtant, en acceptant de convertir la dette en travail servile, Bulalu offre à Yaršar-da'i la certitude d'être nourri tous les jours, et il ménage en même temps ses propres intérêts puisqu'il va disposer d'une main-d'œuvre durable. Durable mais pas irrévocable car Yašar-da'i pourra se libérer en livrant un esclave à Bulalu. Cette faculté de rachat est typique des contrats conclus en période de détresse, pour distinguer les personnes réduites en servitude définitive des débiteurs temporairement ruinés par les crises. La dernière phrase ( Mais s'il prend un esclave pour quelqu'un d'autre, cette tablette le confondra ») laisse d'ailleurs supposer que Yaršar-da'i a d'autres créanciers, et s'est retrouvé pris dans une spirale de surendettement. Parce que Bulalu lui a sauvé la vie, il devient créancier privilégié et passera avant tous les autres.

La famille sert donc de modèle pour créer des liens qui vont au-delà des simples rapports d'obligation entre débiteurs et créanciers. Il y a une remotivation presque affective de la relation juridique, et peut-être même une forme de sentiments charitables entre les parties. Chacun concède quelque chose à l'autre, qui ne fait pas partie du contrat initial : souvent le créancier renonce à une partie de son argent et le débiteur, de son côté, accepte de travailler pour le compte du créancier et de venir s'installer chez lui. Cette communauté de vie qui s'installe entre eux est calquée sur le fonctionnement de la famille, c'est pourquoi elle est formalisée au moyen de contrats comme l'adoption ou la domesticité, qui traduisent un glissement du droit des obligations vers le droit de la famille.

Dans toutes ces situations, ce qui frappe est l'absence d'intervention des pouvoirs publics : la vieillesse, l'isolement, la précarité, ne sont pas pris en charge par l'État mais par les proches ou la collectivité (Stol et Vleeming 1998). Les constructions juridiques 
imaginées par les familles ou les notaires sont quelques fois acrobatiques mais toujours ingénieuses, et à coup sûr efficaces d'où sans doute l'effacement de l'autorité royale, les questions sociales étant mieux prises en charge par les solidarités locales que par les normes générales. Mais il faut souligner que ces initiatives s'analysent comme des libéralités, et sont donc laissées au bon vouloir des individus tant pour leurs modalités que pour leur existence même.

Faut-il alors opposer la passivité étatique à l'« activisme » de terrain? Peut-être pas car l'idéologie royale et la pratique juridique répondent, chacune à leur manière, à une pression sociale forte qui exige à la fois des réactions officielles et des moyens pragmatiques pour préserver l'institution familiale. Il y va du prestige des souverains et de la réputation des élites, principaux acteurs et producteurs de la documentation écrite qui nous est parvenue.

\section{BIBLIOGRAPHIE}

Anbar M. et Stol M. (1991), " Textes de l'époque babylonienne ancienne », Revue d'assyriologie et d'archéologie orientale 85, p. 13-48.

Arnaud D. (1986), Recherches au pays d'Aštata, Emar VI-3, Paris.

Briquel-Chatonnet F. (2010), « Une jeune fille changée en jeune homme : homélie sur un miracle survenu dans le monastère couvent de Qartmin, dans le Tur Abdin ", dans Amir-Moezzi M. A. et al . éd., Pensée grecque et sagesse d'Orient. Hommage à Michel Tardieu, Bibliothèque de l'École des Hautes Études Sciences Religieuses 142, Turnhout, p. 133-152.

Cardascia G. (1959), «L'adoption matrimoniale à Babylone et à Nuzi », RD 37, p. 1-16.

- (1969), Les lois assyriennes, Littératures Anciennes du Proche-Orient 2, Paris.

- (1970), «Adoption matrimoniale et lévirat dans le droit d'Ugarit », Revue d'assyriologie et

d'archéologie orientale 64-2, p. 119-126.

Charpin, D. (2013), «L'historien de la Mésopotamie et ses sources : autour du Code de Hammurabi », Journal asiatique 301-2, p. 339-366.

Démare-Lafont S. (1999), Femmes, droit et justice dans l'Antiquité orientale. Contribution à l'étude du droit pénal au Proche-Orient ancien, Orbis Biblicus et Orientalis 165, Fribourg-Göttingen.

- (2011), « Le juge, gardien des bonnes mœurs en Mésopotamie », dans Chamocho M.A. éd., Droit et Mœurs. Implication et influence des mœurs dans la configuration du droit, Jaén, p. 413-426.

- (2012), « La juridiction domestique au Proche-Orient ancien », dans Otis-Cour L. éd. Histoires de famille. À la convergence du droit pénal et des liens de parenté, Cahiers de l'Institut d'Anthropologie juridique 33, Limoges, p. 55-77.

- (2014), « Modèle familial et solidarités sociales à Émar », dans Marti L. éd., La famille dans le Proche-Orient ancien : réalités, symbolismes et images, Proceedings of the 55th Rencontre Assyriologique Internationale at Paris 6-9 July 2009, Winona Lake, p. 397-412. 
- (2015), « Gender », dans Strawn B.A. éd., The Oxford Encyclopedia of the Bible and Law 1, Oxford, p. 297-306.

Edzard D. O. (1997), Gudea and His Dynasty, The Royal Inscriptions of Mesopotamia Early Periods 3-1, Toronto.

Frayne D. (2007), Presargonic Period (2700-2350 BC), The Royal Inscriptions of Mesopotamia Early Periods 1 , Toronto.

Huehnergard J. (1983), «Five Tablets from the Vicinity of Emar », Revue d'assyriologie et d'archéologie orientale 77, p. 11-43.

Justel J. (2008), « Adoption matrimoniale à Emar », RD 86, p. 1-13.

Lion B. (2007), « La notion de genre en assyriologie », dans Sebillotte Cuchet V. et Ernoult N. éd., Problèmes du genre en Grèce ancienne, Paris, p. 51-64.

- (2009), « Sexe et genre (1) : des filles devenant fils dans les contrats de Nuzi et d'Emar », Topoi Supplément 10, p. 9-25.

- (2011), «Literacy and gender ", dans Radner K. et Robson E. éd., Oxford Handbook of Cuneiform Culture, Oxford, p. 90-112.

Parayre D. (1997), « Les âges de la vie dans le répertoire figuratif oriental », Ktema 22, p. 59-89.

Roth M. (1979), Scholastic Tradition and Mesopotamian Law: A Study of FLP 1287, A Prism in the Collection of the Free Library of Philadelphia, University Microfilms, Ann Arbor.

- (1991), «The Neo-Babylonian Widow », Journal of Cuneiform Studies 43-45, p. 1-26.

- (1997), Law Collections from Mesopotamia and Asia Minor, $2^{\mathrm{e}}$ éd., Writings from the Ancient World - Society of Biblical Literature 6, Atlanta.

Stol M. (2012), " Payment of the Old Babylonian Brideprice », dans Abraham K. et Fleishman J. éd., Looking at the Ancient Near East and the Bible through the Same Eyes. Minha LeAhron: A Tribute to Aaron Skaist, Bethesda, p. 131-167.

- (2016), Women in the Ancient Near East, Boston-Berlin.

Stol M. et Vleeming S. P. éd. (1998), The Care of the Elderly in the Ancient Near East, Studies in the History and Culture of the Ancient Near East 14, Leiden.

Stone E.C. et Owen D. I. (1991), Adoption in Old Babylonian Nippur and the Archive of Mannum-mešu-li șsur, Mesopotamian Civilization 3, Winona Lake.

Tsukimoto A. (1991), « Akkadian Tablets in the Hirayama Collection (II) », Acta Sumerologica 13, p. 275-311.

Westbrook R. (1988), Old Babylonian Marriage Law, Archiv für Orientforschung Beiheft 23, Horn.

- (1995), « Slave and Master in Ancient Near Eastern Law », Chicago-Kent Law Review 70-4, p. $1631-1676$.

- (2000), « International Law in the Amarna Age » dans Cohen R. et Westbrook R. éd., Amarna Diplomacy. The Beginnings of International Relations, Baltimore-Londres, p. 28-41 et 239-242.

Zgoll A. (2013), « Fundamente des Lebens. Vom Potential altorientalischer Mythen », dans Zgoll A. et Kratz R. G. éd., Arbeit am Mythos. Leistung und Grenze des Mythos in Antike und Gegenwart, Tübingen, p. 79-107. 


\section{RÉSUMÉS}

La notion de politique familiale semble absente des sources du Proche-Orient ancien, bien que la famille soit indéniablement une réalité essentielle de la vie sociale et juridique. Cette situation est peut-être due au faible poids de l'autorité publique dans le champ de la vie privée, où dominent plutôt des mécanismes privés de solidarité et de régulation.

The notion of family policy seems to be absent from the Ancient Near Eastern sources, although the family was definitely a crucial element of social and legal life. This situation could stem from the rather limited weight of public authorities in the realm of private life, where private mechanisms of solidarity and regulation prevailed.

\section{INDEX}

Keywords : Mesopotamia, Bible, widow, orphan, royal safeguarding, familial solidarity, will, adoption, voluntary servitude

Mots-clés : Mésopotamie ; Bible ; veuve, orphelin, sauvegarde royale, solidarités familiales, testament, adoption, servitude volontaire

\section{AUTEUR}

SOPHIE DÉMARE-LAFONT

Université Panthéon-Assas, EPHE-PSL - sophie.demare-lafont@u-paris2.fr 